\title{
SIMPLE MODEL DESCRIPTION OF CHEMICAL REACTIVITY AND REACTION PATHWAYS
}

\author{
E. BroclaWIK * \\ Institute of Catalysis and Surface Chemistry, Polish Academy of Sciences \\ Kraków, Poland \\ (Received August 1, 1990)
}

\begin{abstract}
Simple model of preliminary selection of possible reaction pathways for the reaction between multiatomic molecules is proposed. Most probable directions of the attack are estimated from plots of the total energy gradient as a function of mutual orientation of reacting molecules for large intermolecular distances. The method is verified for the oxidation reactions of ethylene and amidogen and also for amidogen interaction with NO. The possibility of the application of the proposed method to catalytic reactions is discussed.
\end{abstract}

PACS numbers: $31.20 .-\mathrm{d}$

\section{Introduction}

Theoretical description of a chemical reaction requires the construction of the potential energy hypersurface for systems composed of reactant molecules for entire manifold of their mutual geometrical arrangements [1-3]. The concept of the potential energy hypersurface for nuclear motions may be introduced basing on the Born-Oppenheimer approximation, fundamental in quantum chemical considèrations. Stationary points on the hypersurface $E(\boldsymbol{R})$ correspond to minima describing stable moieties (substrates, products and intermediates) and to maxima and saddle points describing transition states. Detailed knowledge of such a hypersurface would allow for defining of reaction pathways, e.g. lines in configurational space which connect minima corresponding to substrates and products and pass through appropriate transition states.

In any given coordinate system a reaction pathway can be defined as the steepest descent path or minimum energy path (MEP) from the transition state

"Mailing address: Zakład Chemii Teoretycznej im. K. Gumińskiego, Uniwersytet Jagielloński, Karasia 3, 30-060 Kraków, Poland. 
down to the products and down to the substrates [4-6]. However, for some reactions, the energy hypersurface may be rather complicated and it may not be immediately obvious from either the structure of the transition state or the orientation of the transition vector whether the transition state connects the desired substrates and products. Thus the rigorous definition of the reaction coordinate and the tracing of the MEP with high accuracy requires sofisticated techniques [7] and is tedious and time consuming task, as for each point along the reaction pathway full optimalization of all transverse coordinates must be performed. On the other hand, already the location of stationary points on the potential energy hypersurface for complicated system of reactants is a tremendous problem and further simplification of the model must be adopted to make the job tractable. Analysis of experimental data suggests that the form of the transition state in a given chemical reaction is predetermined already at the preliminary stage of the reaction e.g. by the shape of the potential energy hypersurface at large distances between reactants. Assuming thus that long range interactions between molecules in different mutual geometrical arrangements are approximately proportional to the activation barriers encountered on their approach in these orientations, the calculation of the total energy of the transition complex may be replaced by the estimation of the interaction of substrates for a fixed, long distance between them.

The calculation of the intermolecular interaction at large separation is itself a difficult problem and there were several approximate schemes introduced of the estimation of these interaction energies. They are mostly based on the separation of electrons of both molecules, e.g. the approximation of the wave function of the system by the product of wave functions of isolated subsystems. Such approximation leads to the concept of molecular electrostatic potential produced by one molecule with which the charge distribution of the other molecule may interact [3]. The problem of the reaction of the two subsystems is here reduced to the analysis of molecular electrostatic potential maps which is particularly adequate for simple ionic attacking agents.

Another approach is the one proposed by Fukui $[8,9]$, where the wave function of the system is represented as a combination of Slater determinants constructed from orbitals of separate subsystems but describing also excited and transferred configurations of the combined system. On this basis the intermolecular interaction energy is decomposed into several terms, such as Coulomb interaction term, exchange interaction term and delócalization stabilization term.

In the present paper an approximate scheme is proposed in which simple maps of the energy gradient along hypothetical reaction pathways are constructed and the most promising directions of the attack of one molecule onto the other one may be estimated.

\section{Method and model calculations}

The energy gradient estimated from the differences of the total energies at two chosen points on the reaction coordinate at relatively long distance between reactants may be taken as the indication of the potential energy barrier encountered on approach from the given direction. On that basis the actual calculations 
may be limited to only these reaction pathways which are characterized by the lowest energy barriers and may be therefore assumed to be most probable.

Such approach has been recently applied to study the catalytic oxidation of hydrocarbons $[10,11]$, the main purpose of this investigation was to find qualitative description of the mechanisni of the reaction in question, in particular of the influence of the geometry of adsorbed species and their activation state on the choice of the reaction route and what follows, catalytic selectivity. As the studied systems were multiatomic molecules (toluene and butene attacked by molecular oxygen), semiempirical scheme had to be adopted for calculations of electronic structures, optimal geometries and energies. Among semiempirical quantum chemical methods MNDO type methods proved to be capable of properly describing molecular geometries and energies on the SCF level [12], therefore calculations were performed by means of MNDO method with automatic geometry optimalization procedure [13].

In order to study the reaction pathways in toluene and butene oxidation reaction, simple energy maps were constructed in such a way that the total energy of the system composed of an oxygen species interacting with the attacked organic molecule was calculated for two distances between these reactants, the geometry of the system being kept frozen. Several trajectories along which oxygen moved around an organic molecule were selected, as well in molecular plane as above the fragments of $\pi$-electronic structure, at the distances of 3.0 and $2.5 \AA$. The estimated value of the energy gradient was plotted as a function of the oxygen position on a given trajectory and the most probable regions of its attack were selected on this basis. The reaction pathways starting from these regions were then the subject of detailed study and the influence of the mutual orientation of the reacting molecules and their electronic state on the mechanism of the oxidation was discussed.

The presented results show clearly that the simple energy gradient plots appear to be a helpful tool in selecting the possible reaction pathways in oxidation of hydrocarbons, however there is no basis for more rigorous comparison of the results with an experiment or other thorough theoretical study. Therefore to verify the applicability of the described assumption, three simple model reactions have been studied here by means of the same semiempirical MNDO method. The reaction of ethylene with oxygen molecule is the model of the reaction of singlet oxygen with olefinic bond whereas reactions of amidogen radical with molecular oxygen or nitrogen oxide are interesting as examples of chemical reactions important in atmospheric processes.

\section{Results and discussion}

\section{1. Ethylene + oxygen molecule}

The reaction of the ${ }^{1} \Delta_{\mathrm{g}}$ oxygen molecule with ethylene has been studied [14, $15]$ as the simplest example of the reaction of singlet oxygen with olefinic bond. 
Its mechanism, however, is still not entirely clear, especially the existence of the perepoxide intermediate or the perepoxide-like transition state remains in question. In order to check probability of different reaction pathways the total energy of the system composed of ${ }^{1} \Delta_{\mathrm{g}}$ oxygen molecule and ethylene was calculated for the intermolecular separation equal to 2.5 and $2.0 \AA$, for four mutual geometrical arrangements given in Fig. 1. The starting geometry of the system was the one<smiles></smiles>

(a)<smiles>C=COC=O</smiles>

(c)<smiles>C=COC=O</smiles>

(b)<smiles>C=CC(=O)C=O</smiles>

(d)

Fig. 1. Models of the concerted addition of the singlet oxygen to ethylene.

optimal for separate molecules and it was kept frozen during the computation of energy maps. The oxygen molecule moved paralelly to the $\mathrm{C}=\mathrm{C}$ bond over the plane of the organic molecule. In all cases the total energy was increasing upon decrease of the distance between the reactants, thus indicating existence of the energy barrier (reaction activation energy). The numerical values of $\Delta E=$ $E(d=2.5 \AA)-E(d=2.0 \AA)$ for the position of the oxygen molecule over the center of olefinic bond are given in Table. It is clearly visible that the mutual arrangement described by model $b$ should be the most favourable and that the reaction pathway starting from such orientation should pass through the lowest energy barrier. Moreover, the value of $\Delta E$, while moving over the $\mathrm{C}=\mathrm{C}$ bond, did not change substantially for cases a-c, whereas in geometry $d$ this value dropped to $1.781 \mathrm{eV}$ over the terminal carbon atom what indicated that oxygen tended to escape from the region of the double bond and that the direct parallel attack of $\mathrm{O}_{2}$ onto ethylene $\pi$ bond should be strongly hindered.

TABLE

Total energy differences $\Delta E=E(d=2.5 \AA)-E(d=2.0 \AA)$ and appropriate reaction barriers for models $a-d$ of oxidation of ethylene (in $\mathrm{eV}$ )

\begin{tabular}{c|c|c}
\hline \hline Model & $\Delta E$ & Reaction barrier \\
\hline $\mathrm{a}$ & 1.306 & 3.046 \\
$\mathrm{~b}$ & 0.673 & 2.014 \\
$\mathrm{c}$ & 1.242 & 3.254 \\
$\mathrm{~d}$ & 2.639 & -
\end{tabular}


In order to check reality of the assumed model entire reaction pathways starting from each chosen geometry a-d have been calculated. The reaction coordinate in cases $\mathrm{a}, \mathrm{b}$ and $\mathrm{d}$ was defined as the distance between the center of $\mathrm{C}=\mathrm{C}$ bond and the center of $\mathrm{O}-\mathrm{O}$ bond; in case $\mathrm{c}$ it was defined as the distance between the center of $\mathrm{C}=\mathrm{C}$ bond and the nearer oxygen atom. All other variables have been optimalized in each point along the reaction path. The values of energy barriers encountered on the chosen reaction pathways are listed in Table. The value of the barrier in the case $d$ is not listed because due to the rotational freedom the oxygen molecule immediately changed its orientation to the one close to the starting point of the case $a$. The barrier for the reaction pathway $b$ is substantially lower than these for other cases, in excellent agreement with the previous predictions [14]. Models $\mathrm{b}$ and $\mathrm{c}$ lead to the same product, namely to the perepoxide intermediate with one oxygen bridging carbon atoms and the second one extended vertically to the CCO coplane. Reaction path a leads to the dioxetane cycloadduct which can next undergo $\mathrm{C}-\mathrm{C}$ and $\mathrm{O}-\mathrm{O}$ bond cleavage decomposing thus to carbonyl compounds.

General remarks regarding the mechanism of the oxidation of olefinic bond may be drawn on the basis of the presented selection of probable reaction pathways. Two types of reaction pathways seem to be accessible in this process, first one leading directly to dioxetane formation and the second one giving perepoxide intermediate. Inspection of the total energies of the output compounds alone leads to the conclusion that the dioxetane is thermodynamically much more stable and that this should be the favourable outcome in the first step of oxidation process. However, when the reaction barrier is considered, perepoxide is recommended as a feasible intermediate, in agreement with previous assumptions [15]. It is worthwhile to point out that the last conclusion follows directly from the simple gradient maps proposed as a preliminary tool in studying chemical reactions.

\section{2. Reactions of amidogen}

The reaction between the amidogen radical $\mathrm{NH}_{2}$ and oxygen is the potential source of $\mathrm{NO}_{x}$ species in the troposphere and has therefore stimulated a considerable amount of research $[16,17]$. On the other hand the reaction of amidogen with NO may be utilized for progressive disappearance of nitrogen oxides in the reaction medium $[18,19]$ due to the rapid reaction of the type

$$
\mathrm{NH}_{2}+\mathrm{NO} \rightarrow \mathrm{N}_{2}+\ldots
$$

About both reactions there are heated controversies as to the existence and character of intermediate adduct complexes formed by the combination of $\mathrm{NH}_{2}$ and attacking species. Thus the study of both reactions within the model presented in this paper has been undertaken. Results of the calculation of the total energy difference $\Delta E=E(d=2.5 \AA)-E(d=2.0 \AA)$ for the attacking agent $\mathrm{O}_{2}$ and NO moving around the amidogen radical are shown in Fig. 2. In Fig. 2(a) the gradient profile is presented for the coplanar movement along the trajectory shown in the 

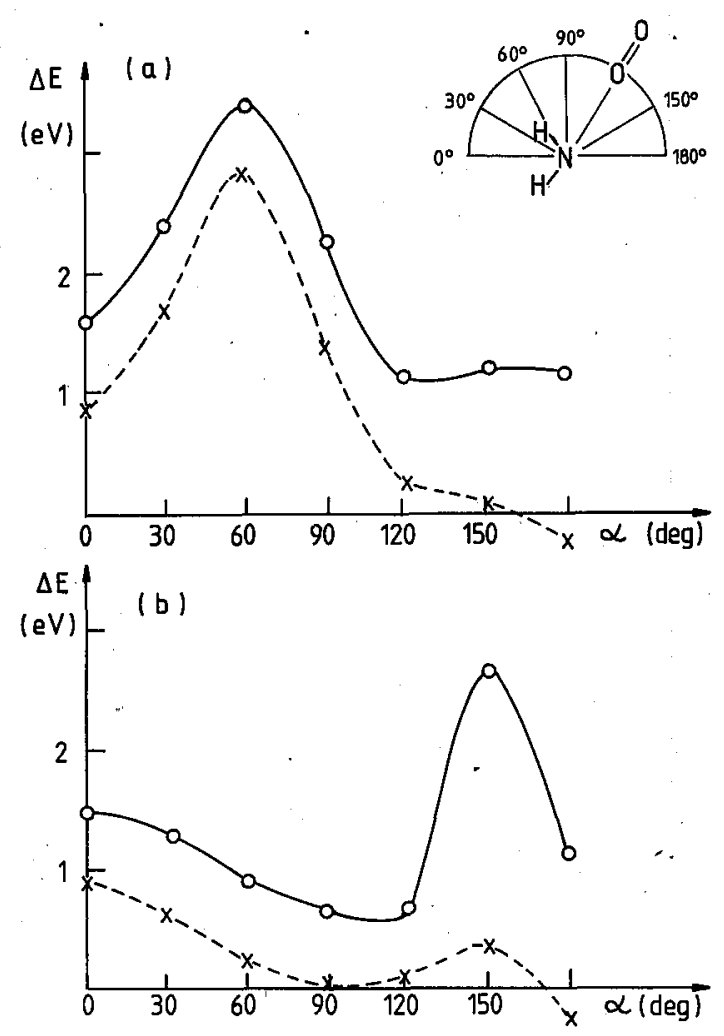

Fig. 2. Total energy gradient plotted as a function of the position of the attacking agent on the trajectory around amidogen radical (full-line $-\mathrm{O}_{2}$, dashed line $-\mathrm{NO}$ ), (a) trajectory in the molecular plane, (b) out-of-plane trajectory.

figure, whereas Fig. 2(b) presents analogous gradient estimates for the out-of-plane movement in the plane bisecting NIII angle (in both pictures the value of the angle equals $0^{\circ}$ as well as $180^{\circ}$ and corresponds to the same position of attacking agent). Other positions of $\mathrm{O}_{2}$ or NO lead to much higher energy barriers.

From the presented plots one can see that for molecular oxygen no regions exist from which the molecule could attack $\mathrm{NH}_{2}$ radical without any energy barrier. The most probable directions of the attack, where the barrier should be relatively low, are the vertical attack on nitrogen atom from above the plane and, slightly less promising, the direction in amidogen plane corresponding to the angle $120^{\circ}$.

The addition reaction involving the recombination of the two highly reactive radicals, $\mathrm{NI}_{2}$ and $\mathrm{NO}$, should obviously proceed without any activation energy. The inspection of curves corresponding to NO in Fig. 2 leads to the conclusion that this is indeed the case for colinear approach of both molecules. There is also large probability of the vertical attack analogous to that of $\mathrm{O}_{2}$ molecule, however only the colinear reaction bears the features of exothermic process with null activation 
barrier.

Similarly as in the case of ethylene oxidation the entire reaction pathways starting from the most promising mutual positions of interacting molecules have been calculated for amidogen reactions. The interaction of $\mathrm{NH}_{2}$ with $\mathrm{NO}$ appeared indeed to be the reactive one and the addition intermediate $\mathrm{NH}_{2} \mathrm{NO}$ was formed with the energy gain of about $1.6 \mathrm{eV}$. The optimal geometrical structure of nitrosamide molecule was in perfect agreement with that found by thorough geometry optimalization with ab inilio 6-31G* calculations [20]. In consequence of considerable amount of available energy the formation of addition compound must proceed in an excited vibrational state. This vibrationally excited adduct can decompose into products on a short time scale by reverse reaction regenerating the reactants or by the intramolecular rearrangement of nitrosamide to hydroxydiimide, $\mathrm{HNNOH}$, apt to further decomposition into $\mathrm{N}_{2}$ and $\mathrm{H}_{2} \mathrm{O}$.

The reaction pathway for the vertical attack of the triplet $\mathrm{O}_{2}$ onto the nitrogen atom leads (with the energy barrier of about $1.2 \mathrm{eV}$ ) to the aminoperoxy radical $\mathrm{NH}_{2} \mathrm{OO}$ with the structure similar to that given by Pouchan [18]. The structure, however, was found not to be coplanar due to the different direction and symmetry of the attack assumed on the basis of the energy maps. The local energy minimum corresponding to $\mathrm{NII}_{2} \mathrm{OO}$ adduct had the energy $0.8 \mathrm{eV}$ higher than the sum of substrates and should undergo further rearrangement leading to nitrous acid. General conclusions may be drawn that the reaction in question requires an activation energy and that its mechanism involving $\mathrm{NII}_{2} \mathrm{OO}$, the addition product of $\mathrm{NII}_{2}$ and $\mathrm{O}_{2}$, is then lighly probable.

\section{Conclusions}

The study of the mechanism of a chemical reaction involving more than one or two atomic reactants is one of the most important tasks of quantum chemistry. It requires knowledge of the potential energy hypersurface for motions of nuclei in the potential of electrons. The calculation of the hypersurface is a tremendous work even with nowadays art of computing. Therefore any information about the shape of some regions of the energy surface allowing to elucidate estimates of the reaction probability is a valuable piece of information especially for large chemical systems.

In the present paper a simple model is presented, within which the promising regions for the attack of one molecule onto the other can be selected in multidimensional space. On this basis the most promising reaction pathways can be selected for further detailed study. Both molecules may be multiatomic moieties and may be examined in different electronic excited states which may provide more information about the system than electrostatic potential maps or charge density investigation of one of the reactants. The method has been applied to model molecular interactions in order to check its validity. The results obtained for the oxidation of ethylene and reactions of amidogen radical seem to indicate that mutual reactivity of the agents and the most favourable orientation of molecules in the preliminary step of the reaction were estimated precisely, in perfect agreement 
with more sofisticated but fragmentary studies. Moreover, some new information could be extracted from the simple gradient maps as to the existence and probability of different routes of the reaction as well as the character of intermediates.

The proposed method applied previously to the oxidation of toluene and butene helped to establish possible geometries of the attack of oxygen onto the organic molecule. Moreover, it enabled the study of the influence of the catalyst -molecule interaction and of mutual geometrical orientation of reactants on the surface, on the catalytic selectivity and the reaction mechanism.

\section{References}

[1] R. Daudel, Quantum Theory of Reactivity, D. Reidel, Dodrecht 1973.

[2] L. Salem, The Molecular Orbilal Theory of Conjugated Systems, W.A. Benjamin, New York 1966.

[3] E. Scrocco, J. Tomasi, Topics in Current Chem. 42, 95 (1973).

[4] K. Fukui, Acc. Chem. Res. 14, 363 (1981).

[5] D.G. Truhlar, B.C. Garret, Acc. Chem. Res. 13, 440 (1980).

[6] W.H. Miller, N.C. Handy, J.E. Adams, J. Chem. Phys. 72, 99 (1980).

[7] K.K. Baldridge, M.S. Gordon, R. Steckler, D.G. Truhlar, J. Phys. Chem. 93, 5107 (1989).

[8] K. Fukui, Topics in Current Chem. 15, 1 (1970).

[9] K. Fukui, Acc. Chem. Res. 21, 448 (1988).

[10] M. Witko, J. Haber, E. Broclawik, in: New Developments in Selective Oxidation, eds. G. Centi, M. Trifiro, Elsevier Sci. Publ., 1990, p. 701.

[11] E. Brocławik, M. Witko, J. Haber, J. Mol. Calal., to be published.

[12] M.J.S. Dewar, H.S. Rzepa, J. Am. Chem. Soc. 100, 58 (1978); ibid. 100, 777 (1978).

[13] M.J.S. Dewar, W. Thiel, J. Am. Chem. Soc. 99, 4899 (1977).

[14] S. Inagaki, S. Yamabe, H. Fujimoto, K. Fukui, Bull. Chem. Soc. Japan 45, 3510 (1972).

[15] A.A. Frimer, Chem. Rev. 79, 359 (1979).

[16] J.N. Crowley, J.R. Sodeau, J. Phys. Chem. 93, 4785 (1989).

[17] H: Abou-Rachid, C. Pouchan, M. Chaillet, Chem. Phys. 90, 243, (1984).

[18] J.N. Crowley, J.R. Sodeau, J. Phys. Chem. 91, 2024 (1987).

[19] C. Pouchan, M. Chaillet, Chem. Phys. Lett. 90, 310 (1982).

[20] J.A. Harrison, R.G.A.R. Maclagan, A.R. Whyte, Chem. Phys. Lett. 130, 98 (1986). 